\title{
Monte Carlo Few-Group Constant Generation for CANDU 6 Core Analysis
}

\author{
Seung Yeol Yoo, Hyung Jin Shim, and Chang Hyo Kim \\ Seoul National University, 1 Gwanak-ro, Gwanak-gu, Seoul 151-744, Republic of Korea \\ Correspondence should be addressed to Hyung Jin Shim; shimhj@snu.ac.kr
}

Received 21 November 2014; Revised 14 January 2015; Accepted 4 February 2015

Academic Editor: Wei Shen

Copyright (C) 2015 Seung Yeol Yoo et al. This is an open access article distributed under the Creative Commons Attribution License, which permits unrestricted use, distribution, and reproduction in any medium, provided the original work is properly cited.

\begin{abstract}
The current neutronics design methodology of CANDU-PHWRs based on the two-step calculations requires determining not only homogenized two-group constants for ordinary fuel bundle lattice cells by the WIMS-AECL lattice cell code but also incremental two-group constants arising from the penetration of control devices into the fuel bundle cells by a supercell analysis code like MULTICELL or DRAGON. As an alternative way to generate the two-group constants necessary for the CANDU-PHWR core analysis, this paper proposes utilizing a $B_{1}$ theory augmented Monte Carlo (MC) few-group constant generation method ( $B_{1}$ MC method) which has been devised for the PWR fuel assembly analysis method. To examine the applicability of the $\mathrm{B}_{1}$ MC method for the CANDU 6 core analysis, the fuel bundle cell and supercell calculations are performed using it to obtain the two-group constants. By showing that the two-group constants from the $\mathrm{B}_{1} \mathrm{MC}$ method agree well with those from WIMS-AECL and that core neutronics calculations for hypothetical CANDU 6 cores by a deterministic diffusion theory code SCAN with $B_{1}$ MC method generated two-group constants also agree well with whole core MC analyses, it is concluded that the $\mathrm{B}_{1} \mathrm{MC}$ method is well qualified for both fuel bundle cell and supercell analyses.
\end{abstract}

\section{Introduction}

A $B_{1}$ theory augmented Monte Carlo (MC) homogenized few-group constant generation method $[1,2]\left(B_{1}\right.$ MC method hereafter) has been proposed as an alternative way to generate homogenized few-group constants of nuclear fuel systems like fuel pins or fuel assemblies or bundles by deterministic fuel assembly spectrum codes like CASMO [3], HELIOS [4], WIMS-AECL [5], and so forth. The applicability of the $B_{1}$ MC method to PWR core analyses has been demonstrated by showing that few-group constants from the method implemented in a Seoul National University (SNU) MC code, McCARD [6] lead to nodal core neutronics calculations in a good agreement with whole PWR core reference MC calculations [2]. The purpose of this paper is to demonstrate that the $\mathrm{B}_{1} \mathrm{MC}$ method is also applicable to the neutronics analysis of CANDU-PHWRs by showing that homogenized two-group constants of fuel bundles of CANDU 6 from it can result in core neutronics calculations that agree very well with a reference CANDU 6 whole core analysis.
As described in detail in [2], the $B_{1}$ MC method generates the homogenized few-group constants of fuel assemblies or fuel bundles in much the same way as its deterministic counterparts [3-5]. Like the latter, the former consists of conducting the infinite medium spectrum (IMS) calculations to determine IMS-weighted homogenized multigroup reaction cross sections of fuel assemblies or fuel bundles, solving multi-group $B_{1}$ equations to determine the critical spectrum (CS) and the critical buckling, and group condensing to obtain the few-group constants including few-group diffusion constants. Unlike the latter, however, the former utilizes continuous energy cross section data available in the evaluated nuclear data libraries and models the geometry of fuel assemblies or bundles exactly as they are instead of using built-in multi-group cross section libraries and approximate modelling of the complex geometry of the fuel assemblies or bundles. Because of these characteristics of treating the nuclear cross section and geometrical data input exactly, the $B_{1}$ MC method may perform inherently more exact IMS calculations, which in turn makes subsequent CS calculations 
conducted on more precise multi-group $B_{1}$ equations, than its deterministic counterparts.

The current neutronics design methodology of CANDUPHWRs is based on the two-step neutronics calculation method represented by lattice cell spectrum codes WIMSAECL [5] and DRAGON [7] and the two-group diffusion theory core analysis code RFSP [8]. The homogenized twogroup constants are obtained by performing the two types of lattice cell computations: the standard unit lattice cell and the supercell calculations. The former is designed to obtain the homogenized two-group constants of a unit lattice cell comprising a fuel bundle, coolant, pressure tube, and associated moderator as a function of reactor state variables, such as temperatures of fuel, moderator, and coolant and fuel depletion, and is done by the WIMS-AECL code. The latter is designed to obtain the incremental cross sections of various control devices such as liquid zone controllers (LZCs), adjuster rods (ADJs), and mechanical control absorbers. The supercell means the standard cell penetrated horizontally or vertically by various control devices. The supercell computations used to be performed by the MULTICELL code [9]. They are currently done by a 3-dimensional collision probability transport theory code, DRAGON, which is developed to tackle the strong heterogeneity posed by the supercell configuration more satisfactorily than the MULTICELL code.

As mentioned previously, the $\mathrm{B}_{1} \mathrm{MC}$ method is inherently advantageous in handling the geometrical heterogeneity and evaluated cross section data without approximation. Therefore, it can serve as an alternative to the two-group constant generation codes for CANDU-PHWR neutronics design, WIMS-AECL and DRAGON. To warrant this qualification, this paper will show how well the two-group constants generated from McCARD [6] by the $\mathrm{B}_{1}$ MC method compare with those from WIMS-AECL. Needless to mention, the comparison of the two-group constants from the $\mathrm{B}_{1} \mathrm{MC}$ and deterministic methods alone is not sufficient to guarantee the qualification of the $\mathrm{B}_{1} \mathrm{MC}$ method or its applicability to neutronics design computations for CANDU-PHWRs. It is prerequisite to show how well the two-group constants from it can predict core neutronics design parameters including the effective multiplication factor $k_{\text {eff }}$ and normalized channel power distribution of CANDU-PHWRs. In order to do so, this paper will perform McCARD whole core transport calculations for three states of a reference CANDU 6 core with all control devices out, a uniform level of LZCs at $50 \%$ fill and all ADJs in. The McCARD whole core analyses for $k_{\text {eff }}$ 's and normalized channel power distributions of the three core states will be compared with the corresponding deterministic neutronics analysis by a SNU diffusion theory code SCAN [10] using the $B_{1}$ MC method-generated two-group constants as inputs. The qualification of the $\mathrm{B}_{1} \mathrm{MC}$ method as the two-group constant generator for two-step neutronics design of CANDU-PHWRs is then demonstrated by showing that deterministic SCAN and the reference McCARD analyses agree well with one another.

Followed by this introduction, the $\mathrm{B}_{1} \mathrm{MC}$ method for both the standard unit lattice cell and the supercell is briefly described in Section 2 to make this paper self-contained. In Section 3, the three hypothetical CANDU 6 core analysis problems are specified against which the qualification of the $\mathrm{B}_{1} \mathrm{MC}$ method as an alternative to the current CANDUPHWR fuel lattice spectrum codes is examined. The $B_{1} M C$ method calculations and WIMS-AECL calculations for the two-group constants of the CANDU 6 lattice cells as well as the CANDU 6 core analyses by SCAN and McCARD are compared in Section 4.

\section{The $B_{1}$ Monte Carlo Method}

A detailed description of the $B_{1}$ MC method is available in $[1,2]$. In order to make this paper self-contained, it is briefly described here. The essential step of the $B_{1}$ MC method involves infinite medium spectrum (IMS) calculations for angular flux $\phi(\mathbf{r}, E, \Omega)$ by the MC neutron transport calculations, which are performed to determine fine-group cross sections of a nuclear fuel system like a fuel assembly or a fuel bundle defined as

$$
\begin{gathered}
\sum_{x, g}=\frac{\int_{V} \int_{\Delta E_{g}} \int_{4 \pi} \Sigma_{x}(\mathbf{r}, E) \phi(\mathbf{r}, E, \boldsymbol{\Omega}) d \mathbf{\Omega} d E d \mathbf{r}}{\int_{V} \int_{\Delta E_{g}} \int_{4 \pi} \phi(\mathbf{r}, E, \boldsymbol{\Omega}) d \boldsymbol{\Omega} d E d \mathbf{r}}, \\
\sum_{s, g^{\prime} g}^{n} \\
=\frac{\int_{V} \int_{\Delta E_{g}} \int_{\Delta E_{g^{\prime}}} \int_{4 \pi} \sum_{s}^{n}\left(\mathbf{r}, E \rightarrow E^{\prime}\right) \phi(\mathbf{r}, E, \boldsymbol{\Omega}) d \mathbf{\Omega} d E^{\prime} d E d \mathbf{r}}{\int_{V} \int_{\Delta E_{g}} \int_{4 \pi} \phi(\mathbf{r}, E, \boldsymbol{\Omega}) d \boldsymbol{\Omega} d E d \mathbf{r}} .
\end{gathered}
$$

$\Sigma_{x, g}(x=$ scattering, absorption, fission $)$ is the $x$-type IMSweighted $g$-group reaction cross section of the nuclear system. $\sum_{s, g^{\prime} g}^{n}(n=0,1)$ is the IMS-weighted $n$th coefficient of Legendre expansion of group transfer scattering cross section. It must be noted that (2) for $\Sigma_{s, g^{\prime} g}^{1}$ derives from an approximation that the energy dependence of the $P_{1}$ component of Legendre expansion of $\phi(\mathbf{r}, E, \boldsymbol{\Omega})$ is proportional to $P_{0}$ component $[1,2]$. Once the IMS-weighted fine-group cross sections in (1) and (2) are obtained through the MC calculations, they are used to specify $\mathrm{B}_{1}$ equations:

$$
\begin{gathered}
\Sigma_{t, g} \phi_{g} \pm i B J_{g}=\sum_{g^{\prime}} \Sigma_{s, g}^{0} \phi_{g^{\prime}}+\chi_{g} \\
\pm i B \phi_{g}+3 \alpha_{g}(B) \Sigma_{t, g} J_{g}=3 \sum_{g^{\prime}} \sum_{s, g g^{\prime}}^{1} J_{g^{\prime}}
\end{gathered}
$$

Like its deterministic counterparts, the $\mathrm{B}_{1} \mathrm{MC}$ method makes use of the solution to the $B_{1}$ equations above, namely, the critical spectrum $\phi_{g}^{B}$, the critical current spectrum $J_{g}^{B}$, and the critical buckling $B_{c}^{2}$, to determine the CS-weighted fewgroup cross sections $\Sigma_{x, G}$ by

$$
\Sigma_{x, G}=\frac{\sum_{g \in G} \Sigma_{x, g} \phi_{g}}{\sum_{g \in G} \phi_{g}}
$$




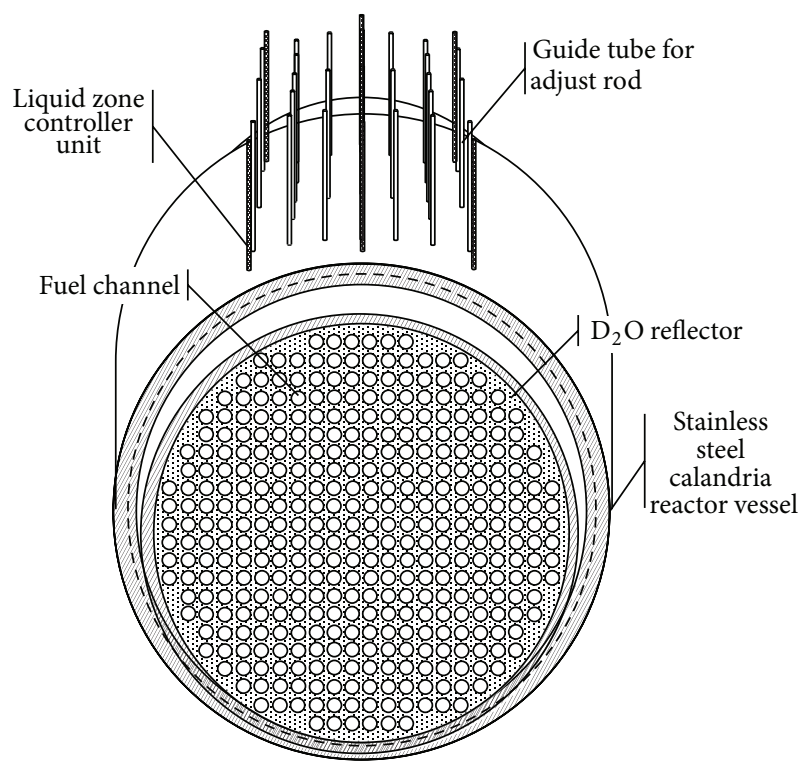

FIGURE 1: Overview of the CANDU 6 core.

and the few-group diffusion constants $D_{G}$ by

$$
D_{G}=\frac{ \pm \sum_{g \in G} i J_{g}^{B}}{\sum_{g \in G} B_{c} \phi_{g}^{B}} .
$$

The $\mathrm{B}_{1}$ MC method has been implemented into the fewgroup generation module of the SNU MC code McCARD [6]. The qualification of the method as a two-group constant generator for the standard unit lattice cell and the supercell will be examined in terms of the CANDU 6 core analysis problems described below.

\section{Hypothetical CANDU 6 Core Analysis Problems}

The hypothetical CANDU 6 reactor has the same geometry and components as the typical CANDU 6 reactor. As shown in Figure 1 , it is composed of the reactor core, the $\mathrm{D}_{2} \mathrm{O}$ reflector, and the stainless steel Calandria reactor vessel. The Calandria is a horizontal cylindrical vessel which envelopes 380 fuel channels comprising the core and contains heavy water moderator and reflector. Eighty out of the 380 fuel channels are depleted ones while the rest are fresh ones. Each fuel channel consists of 12 fuel bundles aligned horizontally inside the pressure tube. Depleted fuel channels contain 2 depleted fuel bundles each, which are positioned at the 3rd and the 4th sites of the 12 fuel bundle sites in the order from the front or end of the fuel channel while all the fresh fuel channels comprise the fresh fuel bundles. The depleted fuel channels are arranged bidirectionally in the center region of the core so that none of two adjacent depleted fuel channels are aligned in the same direction. All the fresh fuel channels are put outside the center region of depleted fuel channels. The core has 4400 fresh fuel bundles and 160 depleted fuel bundles loaded into 380 fuel channels. Figure 2 shows a cross
TABLE 1: Comparison of two-group constants generated by McCARD and WIMS-AECL for the natural uranium fuel bundle.

\begin{tabular}{lccc}
\hline $\begin{array}{l}\text { Two-group } \\
\text { constants }\end{array}$ & McCARD & WIMS-AECL & Rel. Diff. $^{2}(\%)$ \\
\hline$\Sigma_{a 1}$ & $1.64 \times 10^{-3}$ & $1.66 \times 10^{-3}$ & 0.72 \\
$\Sigma_{a 2}$ & $3.48 \times 10^{-3}$ & $3.56 \times 10^{-3}$ & 2.16 \\
$v \Sigma_{f 1}$ & $8.88 \times 10^{-4}$ & $8.95 \times 10^{-4}$ & 1.20 \\
$v \Sigma_{f 2}$ & $4.30 \times 10^{-3}$ & $4.38 \times 10^{-3}$ & 1.84 \\
$\Sigma_{s 12}$ & $8.62 \times 10^{-3}$ & $8.88 \times 10^{-3}$ & 1.66 \\
$\Sigma_{s 21}$ & $6.60 \times 10^{-5}$ & $6.09 \times 10^{-5}$ & -7.73 \\
$D_{1}$ & 1.37 & 1.36 & -0.81 \\
$D_{2}$ & 0.87 & 0.87 & -0.65 \\
\hline
\end{tabular}

${ }^{1}$ Group $1\left(>0.625 \times 10^{-06} \mathrm{MeV}\right)$, group $2\left(<0.625 \times 10^{-06} \mathrm{MeV}\right)$.

${ }^{2}$ Rel. Diff: relative difference between McCARD and WIMS-AECL.

TABLE 2: Comparison of two-group constants generated by McCARD and WIMS-AECL for the depleted uranium fuel bundle.

\begin{tabular}{lccc}
\hline $\begin{array}{l}\text { Two-group } \\
\text { constants }\end{array}$ & McCARD & WIMS-AECL & Rel. Diff. ${ }^{2}(\%)$ \\
\hline$\Sigma_{a 1}$ & $1.60 \times 10^{-3}$ & $1.56 \times 10^{-3}$ & -2.07 \\
$\Sigma_{a 2}$ & $3.14 \times 10^{-3}$ & $3.13 \times 10^{-3}$ & -0.41 \\
$v \Sigma_{f 1}$ & $7.97 \times 10^{-4}$ & $7.93 \times 10^{-4}$ & -0.50 \\
$v \Sigma_{f 2}$ & $3.36 \times 10^{-3}$ & $3.32 \times 10^{-3}$ & -1.21 \\
$\Sigma_{s 12}$ & $8.85 \times 10^{-3}$ & $8.83 \times 10^{-3}$ & -0.19 \\
$\Sigma_{s 21}$ & $5.46 \times 10^{-5}$ & $5.66 \times 10^{-5}$ & 3.66 \\
$D_{1}$ & 1.37 & 1.36 & -0.81 \\
$D_{2}$ & 0.87 & 0.87 & -0.65 \\
\hline
\end{tabular}

${ }^{1}$ Group $1\left(>0.625 \times 10^{-06} \mathrm{MeV}\right)$, group $2\left(<0.625 \times 10^{-06} \mathrm{MeV}\right)$.

${ }^{2}$ Rel. Diff: relative difference between McCARD and WIMS-AECL.

sectional view of the core indicating the positions of the depleted fuel channels.

In order to validate the effectiveness of the $B_{1} \mathrm{MC}$ method for the incremental two-group constant generation, three different states of the hypothetical CANDU such as (i) all-thecontrol-devices-free core, (ii) a core with all the LZCs filled with $50 \%$ water, and (iii) a core with all the ADJs positioned inside the designed locations in the core. Figure 3 shows locations of 14 LZCs which are lumped into 3 types: type 1 representing $6 \mathrm{LZCs}$ in regions $1,4,6,8,11$, and 13, type 26 LZCs in 2, 5, 7, 9, 12, and 14, and type 3 the remaining 2 LZCs in regions 3 and 10. In the LZC core model, the water level of each LZC is set to $50 \%$. Figure 4 shows the core configuration in which all the six types of ADJs are inserted.

The hypothetical cores are presumed to be at hot full power condition with fresh fuels, no xenon and no poisoning material in moderator. Regional temperatures are set to 960.2 $\mathrm{K}$ at fuel, $561.2 \mathrm{~K}$ at coolant and cladding, and $342.2 \mathrm{~K}$ at moderator and structure material. These problems call for determining the $k_{\text {eff }}$ and the normalized channel power distribution. 


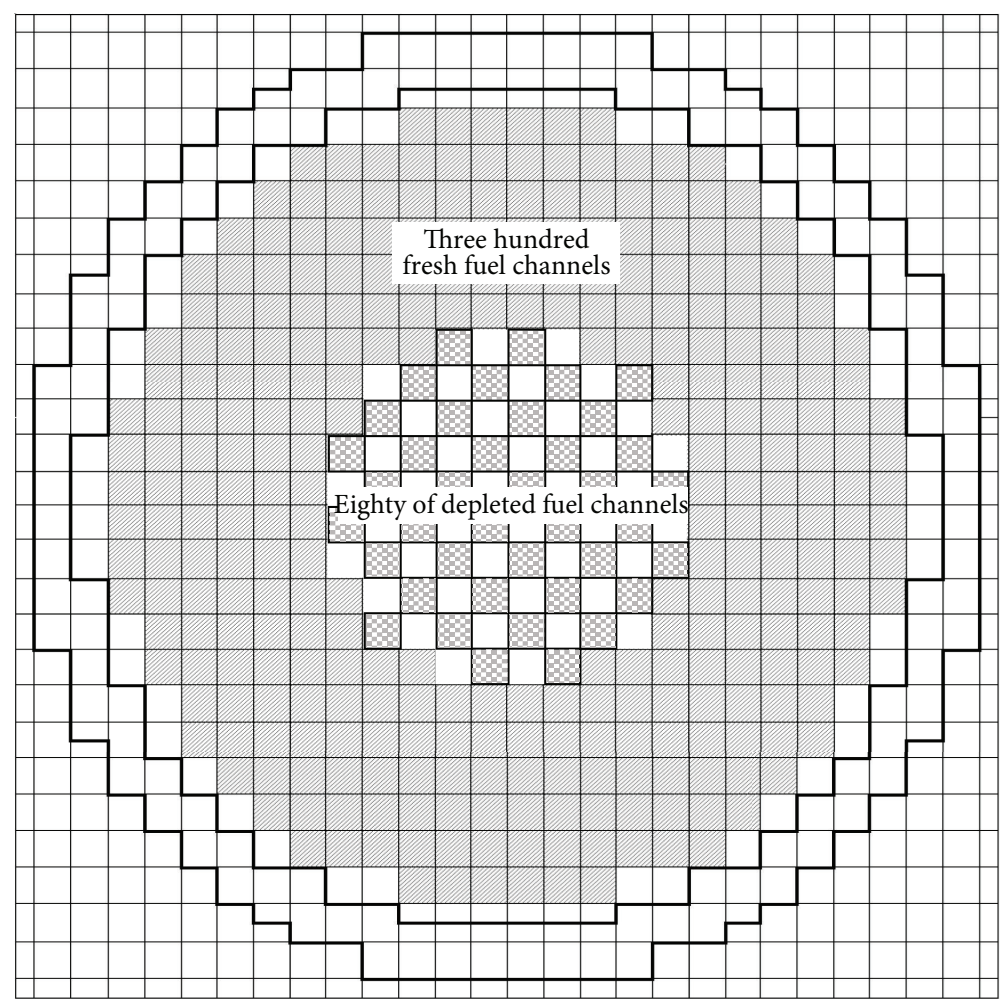

FIgURE 2: Position of the depleted fuel channels.

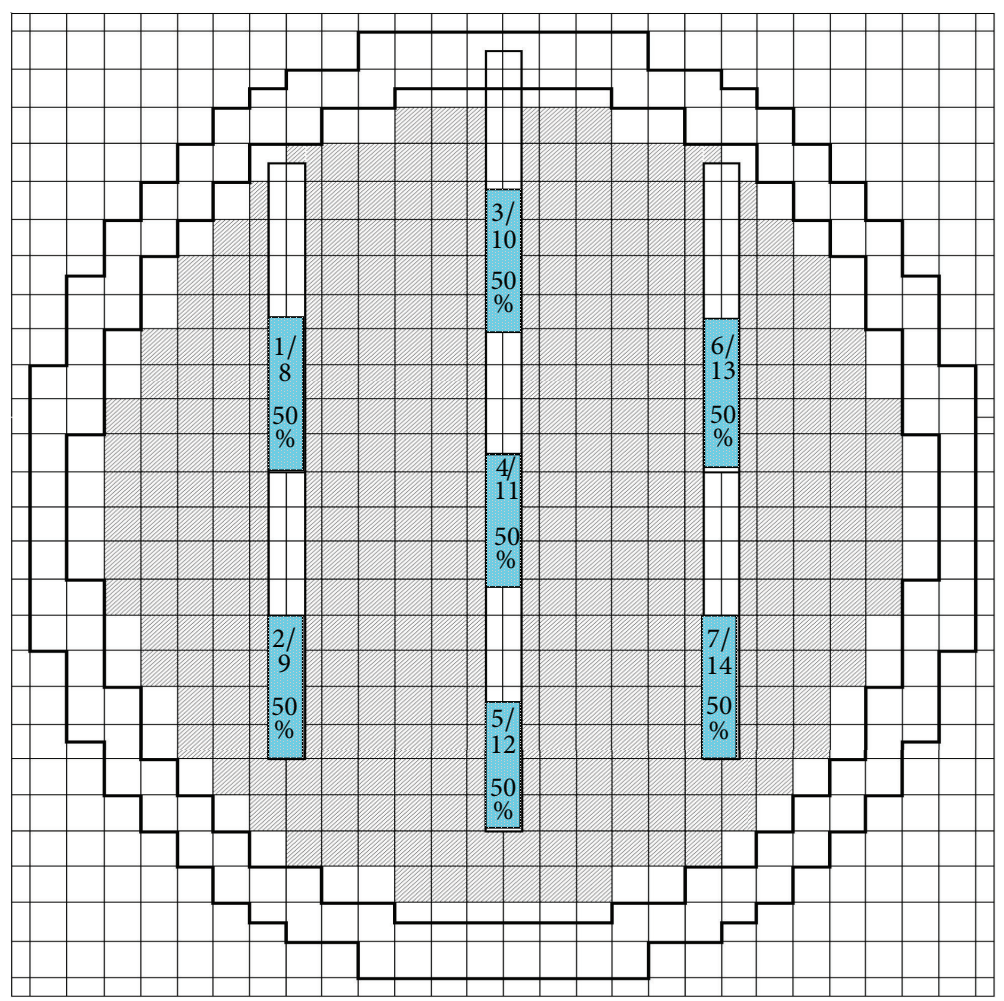

FIGURE 3: Front view of the CANDU 6 core showing LZCs. 


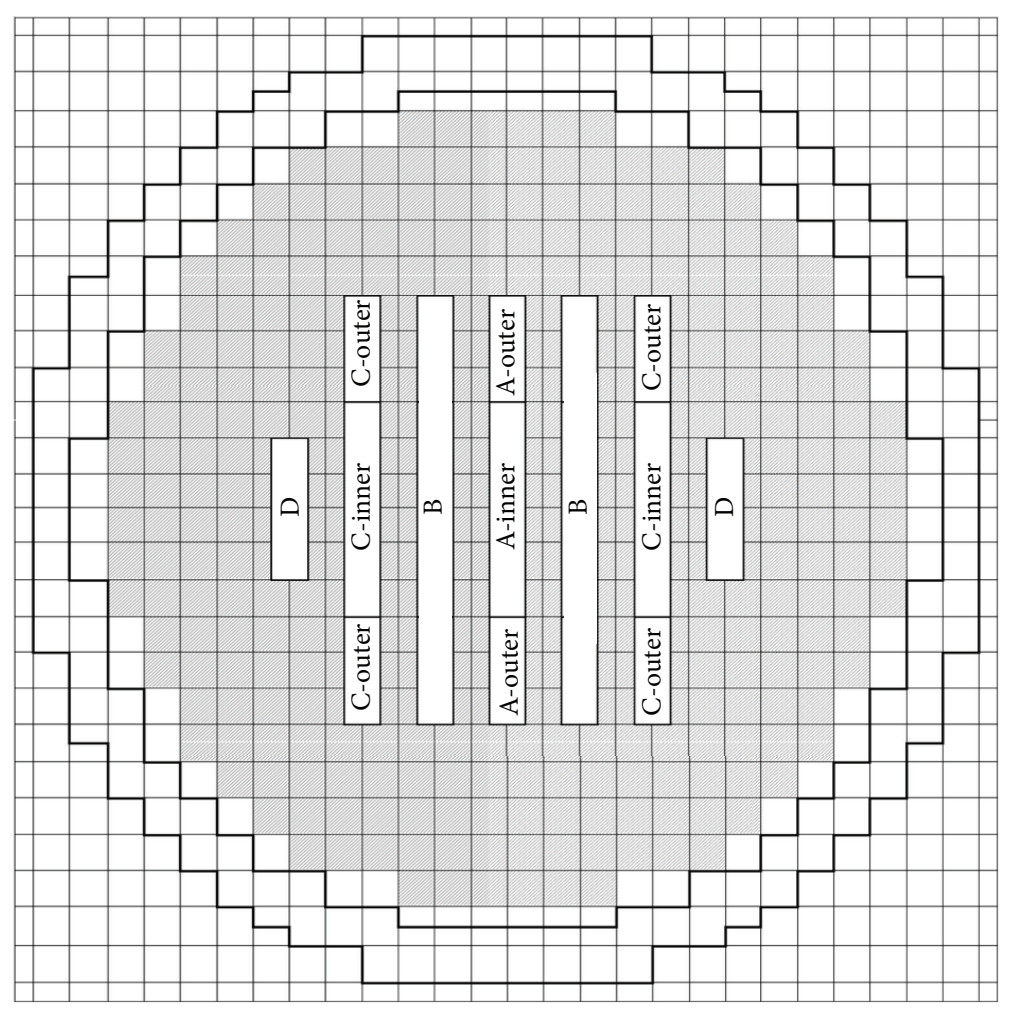

FIgURE 4: Front view of the CANDU 6 core showing ADJs.

TABLE 3: Incremental cross sections of liquid zone controllers.

\begin{tabular}{|c|c|c|c|c|c|c|c|c|}
\hline & \multirow{2}{*}{ Device-free } & & \multicolumn{6}{|c|}{ Device type } \\
\hline & & & Air-1 & $\mathrm{H}_{2} \mathrm{O}-1$ & Air-2 & $\mathrm{H}_{2} \mathrm{O}-2$ & Air-3 & $\mathrm{H}_{2} \mathrm{O}-3$ \\
\hline$\Sigma_{\operatorname{tr} 1}$ & $2.4 \times 10^{-01}$ & $\Delta \Sigma_{\operatorname{tr} 1}$ & $-1.2 \times 10^{-02}$ & $1.5 \times 10^{-02}$ & $-1.4 \times 10^{-02}$ & $1.7 \times 10^{-02}$ & $-9.0 \times 10^{-03}$ & $1.2 \times 10^{-02}$ \\
\hline$\Sigma_{\text {tr } 2}$ & $3.8 \times 10^{-01}$ & $\Delta \Sigma_{\text {tr } 2}$ & $-1.6 \times 10^{-02}$ & $1.2 \times 10^{-01}$ & $-2.5 \times 10^{-02}$ & $1.4 \times 10^{-01}$ & $-7.1 \times 10^{-03}$ & $1.1 \times 10^{-01}$ \\
\hline$\Sigma_{a 1}$ & $1.6 \times 10^{-03}$ & $\Delta \Sigma_{a 1}$ & $-6.1 \times 10^{-06}$ & $7.3 \times 10^{-05}$ & $-1.5 \times 10^{-05}$ & $8.3 \times 10^{-05}$ & $2.7 \times 10^{-06}$ & $6.3 \times 10^{-05}$ \\
\hline$\Sigma_{a 2}$ & $3.5 \times 10^{-03}$ & $\Delta \Sigma_{a 2}$ & $1.7 \times 10^{-04}$ & $1.0 \times 10^{-03}$ & $9.2 \times 10^{-05}$ & $1.1 \times 10^{-03}$ & $2.5 \times 10^{-04}$ & $9.0 \times 10^{-04}$ \\
\hline$v \Sigma_{f 1}$ & $8.9 \times 10^{-04}$ & $\Delta v \Sigma_{f 1}$ & $-2.1 \times 10^{-05}$ & $7.5 \times 10^{-05}$ & $-3.4 \times 10^{-05}$ & $8.8 \times 10^{-05}$ & $-1.0 \times 10^{-05}$ & $6.3 \times 10^{-05}$ \\
\hline$v \Sigma_{f 2}$ & $4.3 \times 10^{-03}$ & $\Delta v \Sigma_{f 2}$ & $2.9 \times 10^{-05}$ & $-1.4 \times 10^{-05}$ & $3.0 \times 10^{-05}$ & $-1.1 \times 10^{-05}$ & $2.6 \times 10^{-05}$ & $-1.4 \times 10^{-05}$ \\
\hline$\Sigma_{s 12}$ & $8.6 \times 10^{-03}$ & $\Delta \Sigma_{s 12}$ & $-2.9 \times 10^{-04}$ & $2.0 \times 10^{-03}$ & $-5.6 \times 10^{-04}$ & $2.3 \times 10^{-03}$ & $-4.5 \times 10^{-05}$ & $1.7 \times 10^{-03}$ \\
\hline$\sum_{s 21}$ & $6.6 \times 10^{-05}$ & $\Delta \Sigma_{s 21}$ & $4.2 \times 10^{-07}$ & $-3.5 \times 10^{-06}$ & $1.5 \times 10^{-06}$ & $-4.4 \times 10^{-06}$ & $-3.9 \times 10^{-07}$ & $-2.8 \times 10^{-06}$ \\
\hline
\end{tabular}

TABLE 4: Incremental cross sections of adjuster rods.

\begin{tabular}{|c|c|c|c|c|c|c|c|c|}
\hline & \multirow{2}{*}{ Device-free } & & \multicolumn{6}{|c|}{ Device type } \\
\hline & & & A-inner & A-outer & B & C-inner & C-outer & $\mathrm{D}$ \\
\hline$\Sigma_{\text {tr } 1}$ & $2.4 \times 10^{-01}$ & $\Delta \Sigma_{\operatorname{tr} 1}$ & $9.0 \times 10^{-04}$ & $8.0 \times 10^{-04}$ & $1.7 \times 10^{-03}$ & $1.6 \times 10^{-03}$ & $6.2 \times 10^{-04}$ & $6.7 \times 10^{-04}$ \\
\hline$\Sigma_{\text {tr } 2}$ & $3.8 \times 10^{-01}$ & $\Delta \Sigma_{\operatorname{tr} 2}$ & $7.3 \times 10^{-04}$ & $6.6 \times 10^{-04}$ & $1.2 \times 10^{-03}$ & $1.2 \times 10^{-03}$ & $5.0 \times 10^{-04}$ & $5.6 \times 10^{-04}$ \\
\hline$\Sigma_{a 1}$ & $1.6 \times 10^{-03}$ & $\Delta \Sigma_{a 1}$ & $2.7 \times 10^{-05}$ & $2.5 \times 10^{-05}$ & $4.7 \times 10^{-05}$ & $4.7 \times 10^{-05}$ & $1.9 \times 10^{-05}$ & $2.0 \times 10^{-05}$ \\
\hline$\Sigma_{a 2}$ & $3.5 \times 10^{-03}$ & $\Delta \Sigma_{a 2}$ & $5.1 \times 10^{-04}$ & $4.6 \times 10^{-04}$ & $8.1 \times 10^{-04}$ & $7.9 \times 10^{-04}$ & $3.7 \times 10^{-04}$ & $4.0 \times 10^{-04}$ \\
\hline$v \Sigma_{f 1}$ & $8.9 \times 10^{-04}$ & $\Delta v \Sigma_{f 1}$ & $-6.5 \times 10^{-06}$ & $-5.6 \times 10^{-06}$ & $-1.0 \times 10^{-05}$ & $-1.0 \times 10^{-05}$ & $-4.6 \times 10^{-06}$ & $-4.8 \times 10^{-06}$ \\
\hline$v \Sigma_{f 2}$ & $4.3 \times 10^{-03}$ & $\Delta v \Sigma_{f 2}$ & $3.5 \times 10^{-05}$ & $3.1 \times 10^{-05}$ & $5.6 \times 10^{-05}$ & $5.5 \times 10^{-05}$ & $2.7 \times 10^{-05}$ & $2.6 \times 10^{-05}$ \\
\hline$\Sigma_{s 12}$ & $8.6 \times 10^{-03}$ & $\Delta \Sigma_{s 12}$ & $1.4 \times 10^{-04}$ & $1.3 \times 10^{-04}$ & $2.1 \times 10^{-04}$ & $2.1 \times 10^{-04}$ & $1.0 \times 10^{-04}$ & $1.1 \times 10^{-04}$ \\
\hline$\Sigma_{s 21}$ & $6.6 \times 10^{-05}$ & $\Delta \Sigma_{s 21}$ & $4.6 \times 10^{-06}$ & $4.2 \times 10^{-06}$ & $7.5 \times 10^{-06}$ & $7.3 \times 10^{-06}$ & $3.3 \times 10^{-06}$ & $3.4 \times 10^{-06}$ \\
\hline
\end{tabular}


TABLE 5: Comparison of $k_{\text {eff }}$ 's for the three core problems.

\begin{tabular}{|c|c|c|c|c|c|}
\hline \multirow[b]{2}{*}{ Core state } & \multirow[b]{2}{*}{ Ref. $^{1} k_{\text {eff }}$} & \multicolumn{2}{|c|}{ McCARD/SCAN (IMS-weighted FGC ${ }^{2}$ ) } & \multicolumn{2}{|c|}{ McCARD/SCAN (CS-weighted FGC) } \\
\hline & & $k_{\mathrm{eff}}$ & Difference $^{3}(\mathrm{pcm})$ & $k_{\mathrm{eff}}$ & Difference $^{3}(\mathrm{pcm})$ \\
\hline No control devices & $1.08709 \pm 0.00001$ & 1.08813 & 104 & 1.08655 & -54 \\
\hline LZCs with $50 \%$ fill & $1.08282 \pm 0.00001$ & 1.08381 & 99 & 1.08220 & -62 \\
\hline All ADJs in & $1.06977 \pm 0.00001$ & 1.07094 & 117 & 1.06934 & -43 \\
\hline
\end{tabular}

${ }^{1}$ Continuous energy McCARD whole core transport calculation.

${ }^{2}$ Few-group constant.

${ }^{3}$ McCARD/SCAN $k_{\text {eff }}-$ Ref. $k_{\text {eff }}$.

\section{Numerical Results and Discussions}

The two-step deterministic solutions to the hypothetical CANDU 6 core analysis problems require specifying the twogroup constants for every 3 -dimensional node. The required two-group constants are produced through the standard unit lattice cell and the supercell calculations by the fewgroup generation module of the McCARD code based on the $B_{1} M C$ method. Figure 5 shows the standard CANDU 6 lattice cell which comprises a 37-element fuel bundle, pressurized heavy-water coolant in a pressure tube, and the associated unpressurized heavy water moderator. Two sets of two-group constants are produced from the standard unit cell calculations with one set with the natural uranium fuel bundle and another with the depleted bundle. Table 1 shows a comparison of the two-group constants from the McCARD calculations and those from the WIMS-AECL Release 2$5 \mathrm{~d}$ calculations for the natural uranium fuel bundle unit cell. Note that the $B_{1} M C$ and deterministic WIMS-AECL calculations produce very similar two-group constants in magnitude. The thermal and fast diffusion constants from the two methods are very close to each other with much less than $1 \%$ relative differences. The two-group reaction cross sections from the two methods are also very similar with about $1 \sim 2 \%$ relative differences. The largest difference between the two is observed in the thermal absorption cross section $\Sigma_{a 2}$ with the relative difference of about $2.2 \%$. Table 2 shows similar comparison of the two-group constants from the $\mathrm{B}_{1} \mathrm{MC}$ and WIMS-AECL calculations for the depleted uranium fuel bundle cell.

To complete the specification of the two-group constants for all nodes of the CANDU 6 core problems, it is necessary to estimate the incremental cross sections representing the effects of the presence of the reactivity devices as well as their guide tubes inside the fuel bundle unit cell on the twogroup constants. The $\mathrm{B}_{1} \mathrm{MC}$ method can estimate them in the same way as the standard unit lattice cell through so-called the supercell model. Figure 6 shows a supercell geometry model adopted in this study for estimating the incremental cross sections arising from the penetration of LZCs or ADJs into the fuel bundle lattice cell. As noted in Figure 6, the dimension of the supercell is 1 lattice pitch $\times 1$ lattice pitch $\times 1$ bundle length, which represents the normal supercell size. The incremental cross sections are computed by the difference of two-group constants of the supercell with and without the guide tube and its control device. Tables 3 and
4 show the $\mathrm{B}_{1} \mathrm{MC}$ method estimates for the incremental cross sections of LZCs and ADJs. The second columns in Tables 3 and 4 list the base two-group constants, $\Sigma_{x}^{\text {base }}$, of the fuel bundle cell without any control devices or guide tubes, for example, LZC and its guide tube in Table 3. The air- $n$ $(n=1,2,3)$ columns in Table 3 list the incremental twogroup constants of type $n$ LZCs with their zone control units filled with air, $\Delta \Sigma_{x}^{\text {air }}$. The $\mathrm{H}_{2} \mathrm{O}-n(n=1,2,3)$ columns list the incremental two-group constants of type $n$ LZCs with their zone control units filled with $\mathrm{H}_{2} \mathrm{O}$.

As shown above, the $B_{1} M C$ method can generate the CSweighted two-group constants of the ordinary fuel bundle cells free from control devices and the incremental two-group constants of the supercell. In addition, the IMS-weighted twogroup constants are generated to investigate the effect of the critical spectrum on the two-group constants. To validate their qualification for the CANDU-PHWR neutronics design calculations, they are used for the core neutronics analysis of the CANDU 6 core problems by the finite difference method option of the SCAN code [10]. The SCAN calculations are conducted in the $84 \times 68 \times 40$ fine mesh model with reflector cross sections given with RFSP-IST version REL 304 and zero flux conditions at extrapolated boundaries in the axial and radial directions. Table 5 shows comparisons of $k_{\text {eff }}$ 's of the three different core states of the hypothetical CANDU 6 core calculated by SCAN with reference solutions obtained by the continuous energy McCARD whole core calculations with 1200 cycles including 200 inactive cycles with 1,000,000 histories per cycle. One can see that $k_{\text {eff }}$ from the SCAN calculations with the CS-weighted two-step constants agrees very well with the reference McCARD whole core predictions. In this conjunction, it is noted that the differences in $k_{\text {eff }}$ 's between SCAN with the CS-weighted twogroup constants and the reference McCARD are $-54 \mathrm{pcm}$ for the no-control-device core, $-62 \mathrm{pcm}$ for the LZC-50\% fill core, and $-43 \mathrm{pcm}$ for the all-ADJ-in core while those from SCAN with the IMS-weighted two-group constants $104 \mathrm{pcm}$, $99 \mathrm{pcm}$, and $117 \mathrm{pcm}$, respectively. Tables 6,7 , and 8 show comparisons of the channel power distributions obtained by folding the full core results in the $1 / 4$ core model for the three different core states with no control devices, LZCs at $50 \%$ fill, and all ADJs in, respectively. From these figures, one can again see that the channel power distributions from McCARD/SCAN two-step calculations agree very well with those from the reference McCARD calculations. Note that the root mean square (RMS) errors of the SCAN predictions with the CS-weighted two-group cross sections to the reference 
TABLE 6: Comparison of normalized channel power distribution for the control-device-free core.

\begin{tabular}{|c|c|c|c|c|c|c|c|c|c|c|}
\hline 1.370 & 1.370 & 1.350 & 1.370 & 1.330 & 1.310 & 1.220 & 1.100 & 0.944 & 0.754 & 0.554 \\
\hline 0.03 & 0.03 & 0.03 & 0.03 & 0.03 & 0.03 & 0.03 & 0.03 & 0.03 & 0.04 & 0.04 \\
\hline-0.11 & -0.14 & -0.07 & -0.14 & -0.18 & -0.11 & -0.14 & -0.09 & -0.28 & -0.40 & 0.11 \\
\hline 0.18 & 0.14 & 0.17 & -0.27 & -0.13 & 0.42 & 0.35 & -0.02 & -0.34 & -0.49 & 0.13 \\
\hline 1.370 & 1.360 & 1.350 & 1.360 & 1.320 & 1.280 & 1.190 & 1.070 & 0.918 & 0.729 & 0.530 \\
\hline 0.03 & 0.03 & 0.03 & 0.03 & 0.03 & 0.03 & 0.03 & 0.03 & 0.03 & 0.04 & 0.04 \\
\hline-0.19 & -0.17 & -0.12 & -0.18 & -0.17 & -0.08 & -0.16 & 0.00 & -0.18 & -0.26 & 0.24 \\
\hline 0.09 & 0.09 & 0.11 & -0.25 & -0.27 & 0.28 & 0.37 & -0.12 & -0.36 & -0.43 & 0.20 \\
\hline 1.350 & 1.350 & 1.330 & 1.340 & 1.340 & 1.240 & 1.140 & 1.020 & 0.867 & 0.682 & 0.496 \\
\hline 0.03 & 0.03 & 0.03 & 0.03 & 0.03 & 0.03 & 0.03 & 0.03 & 0.03 & 0.04 & 0.05 \\
\hline-0.22 & -0.14 & -0.17 & -0.14 & -0.06 & -0.06 & -0.16 & -0.14 & -0.16 & 0.12 & 0.85 \\
\hline 0.02 & 0.09 & 0.03 & -0.33 & -0.29 & 0.20 & 0.28 & -0.26 & -0.43 & -0.09 & 0.83 \\
\hline 1.330 & 1.330 & 1.310 & 1.310 & 1.290 & 1.180 & 1.060 & 0.950 & 0.795 & 0.625 & \\
\hline 0.03 & 0.03 & 0.03 & 0.03 & 0.03 & 0.03 & 0.03 & 0.03 & 0.04 & 0.04 & \\
\hline-0.20 & -0.18 & -0.17 & -0.21 & -0.02 & 0.05 & -0.03 & 0.02 & 0.04 & 0.73 & \\
\hline 0.00 & 0.01 & -0.03 & -0.36 & -0.32 & 0.23 & 0.32 & -0.32 & -0.21 & 0.56 & \\
\hline 1.310 & 1.300 & 1.330 & 1.310 & 1.230 & 1.100 & 0.980 & 0.861 & 0.707 & 0.548 & \\
\hline 0.03 & 0.03 & 0.03 & 0.03 & 0.03 & 0.03 & 0.03 & 0.03 & 0.04 & 0.04 & \\
\hline-0.20 & -0.15 & -0.07 & -0.04 & 0.02 & -0.02 & -0.04 & 0.12 & 0.44 & 1.34 & \\
\hline-0.07 & -0.05 & -0.05 & -0.24 & -0.36 & 0.16 & 0.28 & -0.39 & 0.06 & 1.18 & \\
\hline 1.310 & 1.300 & 1.270 & 1.230 & 1.140 & 1.010 & 0.884 & 0.756 & 0.612 & & \\
\hline 0.03 & 0.03 & 0.03 & 0.03 & 0.03 & 0.03 & 0.03 & 0.04 & 0.04 & & \\
\hline 0.02 & 0.02 & -0.02 & -0.09 & -0.06 & -0.03 & 0.02 & 0.14 & 0.93 & & \\
\hline 0.00 & -0.01 & -0.08 & -0.31 & -0.28 & 0.20 & 0.27 & -0.21 & 0.72 & & \\
\hline 1.220 & 1.210 & 1.170 & 1.120 & 1.030 & 0.903 & 0.771 & 0.637 & 0.499 & & \\
\hline 0.03 & 0.03 & 0.03 & 0.03 & 0.03 & 0.03 & 0.04 & 0.04 & 0.05 & & \\
\hline-0.14 & -0.23 & -0.19 & -0.19 & -0.14 & -0.16 & -0.13 & 0.41 & 1.39 & & \\
\hline-0.23 & -0.32 & -0.30 & -0.43 & -0.40 & 0.03 & 0.22 & 0.21 & 1.24 & & \\
\hline 1.100 & 1.070 & 1.030 & 0.977 & 0.887 & 0.769 & 0.640 & 0.520 & & & \\
\hline 0.03 & 0.03 & 0.03 & 0.03 & 0.03 & 0.04 & 0.04 & 0.05 & & & \\
\hline-0.14 & -0.09 & -0.13 & -0.11 & -0.13 & -0.04 & 0.32 & 0.78 & & & \\
\hline-0.26 & -0.22 & -0.26 & -0.34 & -0.33 & -0.05 & 0.37 & 0.86 & & & \\
\hline 0.929 & 0.908 & 0.865 & 0.806 & 0.719 & 0.610 & 0.504 & & & & \\
\hline 0.03 & 0.03 & 0.03 & 0.04 & 0.04 & 0.04 & 0.05 & & & & \\
\hline-0.13 & -0.14 & -0.12 & 0.01 & -0.04 & 0.32 & 1.00 & & & & \\
\hline-0.26 & -0.27 & -0.25 & -0.16 & -0.20 & 0.23 & 0.96 & & & & \\
\hline 0.736 & 0.715 & 0.674 & 0.627 & 0.544 & 0.449 & & & & & \\
\hline 0.04 & 0.04 & 0.04 & 0.04 & 0.04 & 0.05 & & & & & \\
\hline 0.03 & 0.04 & 0.33 & 0.67 & 0.57 & 1.10 & & & & & \\
\hline-0.08 & -0.07 & 0.25 & 0.71 & 0.72 & 1.00 & & & & & \\
\hline 0.539 & 0.517 & 0.487 & & & & & & & $P_{\text {Ref }}$ & \\
\hline 0.04 & 0.05 & 0.05 & & & & & & & Rel. S.D. (\%) & \\
\hline 1.24 & 1.23 & 2.22 & & & & & & & Diff. $_{\text {INF. }}{ }^{*}$ & \\
\hline 1.26 & 1.26 & 2.33 & & & & & & & Diff. $_{\text {CRI }}{ }^{* *}$ & \\
\hline
\end{tabular}

$P_{\text {Ref }}=$ McCARD power, Rel. S.D. $(\%)=$ relative standard deviation of $P_{\text {Ref }}$.

$P_{\mathrm{INF}}=$ McCARD/SCAN power by infinite medium spectrum.

$P_{\mathrm{CRI}}=\mathrm{McCARD} / \mathrm{SCAN}$ power by critical spectrum.

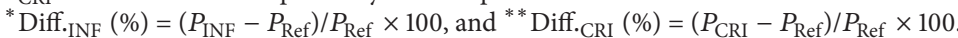


TABLE 7: Comparison of normalized channel power distribution for the core with LZCs at $50 \%$ fill.

\begin{tabular}{|c|c|c|c|c|c|c|c|c|c|c|}
\hline 1.367 & 1.391 & 1.392 & 1.369 & 1.33 & 1.309 & 1.22 & 1.102 & 0.944 & 0.754 & 0.554 \\
\hline 0.03 & 0.03 & 0.03 & 0.03 & 0.03 & 0.03 & 0.03 & 0.03 & 0.03 & 0.04 & 0.04 \\
\hline-0.34 & -0.14 & -0.06 & 0.12 & 0.12 & 0.27 & 0.12 & 0.17 & 0.07 & 0.04 & 0.53 \\
\hline-0.08 & 0.06 & 0.12 & 0.27 & 0.21 & 0.26 & 0.04 & 0.04 & -0.07 & -0.09 & 0.53 \\
\hline 1.371 & 1.387 & 1.384 & 1.36 & 1.317 & 1.283 & 1.188 & 1.074 & 0.917 & 0.73 & 0.531 \\
\hline 0.03 & 0.03 & 0.03 & 0.03 & 0.03 & 0.03 & 0.03 & 0.03 & 0.03 & 0.04 & 0.04 \\
\hline-0.23 & -0.02 & 0.04 & 0.05 & 0.05 & 0.14 & -0.01 & 0.08 & 0.07 & -0.03 & 0.50 \\
\hline 0.02 & 0.17 & 0.21 & 0.19 & 0.12 & 0.16 & -0.05 & -0.06 & -0.08 & -0.15 & 0.51 \\
\hline 1.371 & 1.378 & 1.368 & 1.342 & 1.334 & 1.241 & 1.136 & 1.022 & 0.868 & 0.683 & 0.497 \\
\hline 0.03 & 0.03 & 0.03 & 0.03 & 0.03 & 0.03 & 0.03 & 0.03 & 0.03 & 0.04 & 0.05 \\
\hline-0.03 & 0.00 & 0.05 & 0.03 & 0.10 & 0.01 & -0.14 & -0.03 & -0.11 & 0.30 & 1.00 \\
\hline 0.16 & 0.18 & 0.20 & 0.13 & 0.11 & 0.01 & -0.18 & -0.16 & -0.24 & 0.21 & 1.09 \\
\hline 1.351 & 1.355 & 1.344 & 1.311 & 1.291 & 1.18 & 1.063 & 0.949 & 0.796 & 0.626 & \\
\hline 0.03 & 0.03 & 0.03 & 0.03 & 0.03 & 0.03 & 0.03 & 0.03 & 0.04 & 0.04 & \\
\hline 0.15 & 0.08 & 0.07 & 0.01 & 0.07 & -0.19 & -0.24 & -0.01 & 0.11 & 0.76 & \\
\hline 0.31 & 0.22 & 0.17 & 0.07 & 0.03 & -0.17 & -0.25 & -0.14 & -0.02 & 0.75 & \\
\hline 1.309 & 1.316 & 1.35 & 1.309 & 1.23 & 1.104 & 0.979 & 0.86 & 0.707 & 0.548 & \\
\hline 0.03 & 0.03 & 0.03 & 0.03 & 0.03 & 0.03 & 0.03 & 0.03 & 0.04 & 0.04 & \\
\hline 0.17 & 0.14 & 0.17 & 0.14 & -0.03 & -0.23 & -0.38 & -0.09 & 0.41 & 1.42 & \\
\hline 0.28 & 0.20 & 0.16 & 0.09 & -0.11 & -0.23 & -0.39 & -0.22 & 0.33 & 1.51 & \\
\hline 1.281 & 1.296 & 1.282 & 1.233 & 1.145 & 1.017 & 0.885 & 0.757 & 0.613 & & \\
\hline 0.03 & 0.03 & 0.03 & 0.03 & 0.03 & 0.03 & 0.03 & 0.04 & 0.04 & & \\
\hline 0.01 & 0.14 & 0.10 & 0.00 & -0.16 & -0.39 & -0.47 & -0.21 & 0.69 & & \\
\hline 0.05 & 0.09 & 0.02 & -0.11 & -0.28 & -0.44 & -0.51 & -0.31 & 0.69 & & \\
\hline 1.176 & 1.194 & 1.176 & 1.121 & 1.032 & 0.906 & 0.773 & 0.638 & 0.500 & & \\
\hline 0.03 & 0.03 & 0.03 & 0.03 & 0.03 & 0.03 & 0.04 & 0.04 & 0.05 & & \\
\hline-0.24 & -0.15 & -0.14 & -0.20 & -0.43 & -0.61 & -0.67 & -0.09 & 0.97 & & \\
\hline-0.22 & -0.25 & -0.26 & -0.33 & -0.56 & -0.68 & -0.72 & -0.16 & 1.06 & & \\
\hline 1.043 & 1.057 & 1.035 & 0.977 & 0.889 & 0.772 & 0.642 & 0.522 & & & \\
\hline 0.03 & 0.03 & 0.03 & 0.03 & 0.03 & 0.04 & 0.04 & 0.05 & & & \\
\hline-0.29 & -0.19 & -0.17 & -0.14 & -0.44 & -0.74 & -0.52 & 0.13 & & & \\
\hline-0.30 & -0.32 & -0.32 & -0.28 & -0.58 & -0.84 & -0.56 & 0.21 & & & \\
\hline 0.888 & 0.893 & 0.865 & 0.807 & 0.721 & 0.612 & 0.506 & & & & \\
\hline 0.03 & 0.03 & 0.03 & 0.04 & 0.04 & 0.04 & 0.05 & & & & \\
\hline-0.31 & -0.19 & -0.14 & -0.16 & -0.41 & -0.61 & -0.14 & & & & \\
\hline-0.35 & -0.32 & -0.28 & -0.29 & -0.52 & -0.66 & -0.05 & & & & \\
\hline 0.715 & 0.707 & 0.676 & 0.629 & 0.545 & 0.45 & & & & & \\
\hline 0.04 & 0.04 & 0.04 & 0.04 & 0.04 & 0.05 & & & & & \\
\hline-0.30 & -0.09 & 0.24 & 0.43 & 0.32 & 0.16 & & & & & \\
\hline-0.38 & -0.20 & 0.16 & 0.41 & 0.34 & 0.28 & & & & & \\
\hline 0.529 & 0.514 & 0.489 & & & & & & & $P_{\text {Ref }}$ & \\
\hline 0.04 & 0.05 & 0.05 & & & & & & & Rel. S.D. (\%) & \\
\hline 0.84 & 1.06 & 2.07 & & & & & & & Diff. $_{\text {INF }}{ }^{*}$ & \\
\hline 0.89 & 1.09 & 2.18 & & & & & & & Diff. $_{\mathrm{CRI}}{ }^{* *}$ & \\
\hline
\end{tabular}

$P_{\text {Ref }}=$ McCARD power, Rel. S.D. $(\%)=$ relative standard deviation of $P_{\text {Ref }}$.

$P_{\mathrm{INF}}=$ McCARD/SCAN power by infinite medium spectrum.

$P_{\mathrm{CRI}}=$ McCARD/SCAN power by critical spectrum.

${ }^{*} \operatorname{Diff}_{\text {INF }}(\%)=\left(P_{\mathrm{INF}}-P_{\mathrm{Ref}}\right) / P_{\mathrm{Ref}} \times 100$, and ${ }^{* *}$ Diff. $_{\text {CRI }}(\%)=\left(P_{\mathrm{CRI}}-P_{\mathrm{Ref}}\right) / P_{\mathrm{Ref}} \times 100$. 
TABLE 8: Comparison of normalized channel power distribution for the core with all ADJs in.

\begin{tabular}{|c|c|c|c|c|c|c|c|c|c|c|}
\hline 0.916 & 0.916 & 0.940 & 0.988 & 1.060 & 1.180 & 1.220 & 1.200 & 1.080 & 0.896 & 0.670 \\
\hline 0.03 & 0.03 & 0.03 & 0.03 & 0.03 & 0.03 & 0.03 & 0.03 & 0.03 & 0.03 & 0.04 \\
\hline-2.55 & -2.29 & -2.01 & -1.57 & -1.20 & -0.56 & -0.17 & 0.26 & 0.36 & 0.42 & 1.02 \\
\hline-1.80 & -1.60 & -1.40 & -1.09 & -0.90 & -0.48 & -0.25 & 0.07 & 0.10 & 0.15 & 0.86 \\
\hline 0.932 & 0.932 & 0.958 & 1.010 & 1.080 & 1.200 & 1.220 & 1.190 & 1.060 & 0.872 & 0.645 \\
\hline 0.03 & 0.03 & 0.03 & 0.03 & 0.03 & 0.03 & 0.03 & 0.03 & 0.03 & 0.03 & 0.04 \\
\hline-2.35 & -2.15 & -1.82 & -1.39 & -1.01 & -0.51 & -0.15 & 0.27 & 0.37 & 0.46 & 0.96 \\
\hline-1.64 & -1.50 & -1.25 & -0.96 & -0.76 & -0.47 & -0.26 & 0.07 & 0.11 & 0.20 & 0.81 \\
\hline 0.965 & 0.966 & 0.993 & 1.040 & 1.160 & 1.230 & 1.230 & 1.170 & 1.030 & 0.826 & 0.606 \\
\hline 0.03 & 0.03 & 0.03 & 0.03 & 0.03 & 0.03 & 0.03 & 0.03 & 0.03 & 0.04 & 0.04 \\
\hline-1.96 & -1.86 & -1.56 & -1.10 & -0.63 & -0.33 & -0.04 & 0.30 & 0.44 & 0.81 & 1.50 \\
\hline-1.34 & -1.29 & -1.07 & -0.76 & -0.51 & -0.34 & -0.17 & 0.08 & 0.18 & 0.57 & 1.43 \\
\hline 1.020 & 1.010 & 1.040 & 1.100 & 1.200 & 1.240 & 1.210 & 1.120 & 0.960 & 0.765 & \\
\hline 0.03 & 0.03 & 0.03 & 0.03 & 0.03 & 0.03 & 0.03 & 0.03 & 0.03 & 0.04 & \\
\hline-1.58 & -1.42 & -1.19 & -0.87 & -0.37 & -0.15 & 0.16 & 0.50 & 0.76 & 1.51 & \\
\hline-1.07 & -0.96 & -0.82 & -0.61 & -0.32 & -0.23 & -0.02 & 0.27 & 0.51 & 1.35 & \\
\hline 1.080 & 1.080 & 1.140 & 1.190 & 1.220 & 1.220 & 1.160 & 1.040 & 0.870 & 0.678 & \\
\hline 0.03 & 0.03 & 0.03 & 0.03 & 0.03 & 0.03 & 0.03 & 0.03 & 0.03 & 0.04 & \\
\hline-1.14 & -0.97 & -0.55 & -0.37 & -0.13 & -0.05 & 0.26 & 0.57 & 1.12 & 2.06 & \\
\hline-0.80 & -0.69 & -0.39 & -0.30 & -0.18 & -0.18 & 0.05 & 0.33 & 0.90 & 2.00 & \\
\hline 1.190 & 1.180 & 1.190 & 1.210 & 1.200 & 1.170 & 1.080 & 0.936 & 0.765 & & \\
\hline 0.03 & 0.03 & 0.03 & 0.03 & 0.03 & 0.03 & 0.03 & 0.03 & 0.04 & & \\
\hline-0.36 & -0.20 & -0.10 & -0.05 & 0.03 & 0.13 & 0.29 & 0.73 & 1.45 & & \\
\hline-0.27 & -0.16 & -0.11 & -0.11 & -0.10 & -0.05 & 0.07 & 0.49 & 1.31 & & \\
\hline 1.240 & 1.220 & 1.210 & 1.190 & 1.150 & 1.070 & 0.956 & 0.798 & 0.627 & & \\
\hline 0.03 & 0.03 & 0.03 & 0.03 & 0.03 & 0.03 & 0.03 & 0.04 & 0.04 & & \\
\hline 0.03 & 0.11 & 0.15 & 0.06 & 0.07 & 0.21 & 0.32 & 0.95 & 1.93 & & \\
\hline-0.02 & 0.06 & 0.07 & -0.06 & -0.09 & 0.00 & 0.09 & 0.75 & 1.89 & & \\
\hline 1.200 & 1.180 & 1.150 & 1.110 & 1.040 & 0.932 & 0.798 & 0.654 & & & \\
\hline 0.03 & 0.03 & 0.03 & 0.03 & 0.03 & 0.03 & 0.04 & 0.04 & & & \\
\hline 0.57 & 0.53 & 0.54 & 0.48 & 0.31 & 0.47 & 0.82 & 1.40 & & & \\
\hline 0.42 & 0.36 & 0.36 & 0.28 & 0.10 & 0.25 & 0.63 & 1.34 & & & \\
\hline 1.080 & 1.060 & 1.020 & 0.954 & 0.865 & 0.750 & 0.629 & & & & \\
\hline 0.03 & 0.03 & 0.03 & 0.03 & 0.03 & 0.04 & 0.04 & & & & \\
\hline 0.73 & 0.70 & 0.70 & 0.71 & 0.51 & 0.78 & 1.31 & & & & \\
\hline 0.53 & 0.49 & 0.49 & 0.50 & 0.30 & 0.60 & 1.26 & & & & \\
\hline 0.892 & 0.867 & 0.821 & 0.762 & 0.665 & 0.553 & & & & & \\
\hline 0.03 & 0.03 & 0.04 & 0.04 & 0.04 & 0.04 & & & & & \\
\hline 0.90 & 0.94 & 1.36 & 1.54 & 1.39 & 1.61 & & & & & \\
\hline 0.69 & 0.74 & 1.18 & 1.42 & 1.31 & 1.61 & & & & & \\
\hline 0.667 & 0.641 & 0.602 & & & & & & & $P_{\text {Ref }}$ & \\
\hline 0.04 & 0.04 & 0.04 & & & & & & & Rel. S.D. (\%) & \\
\hline 2.14 & 2.15 & 3.23 & & & & & & & Diff. $_{\text {INF }}^{*}$ & \\
\hline 2.06 & 2.08 & 3.25 & & & & & & & Diff. $_{\mathrm{CRI}}{ }^{* *}$ & \\
\hline
\end{tabular}

$P_{\text {Ref }}=$ McCARD power, Rel. S.D. $(\%)=$ relative standard deviation of $P_{\text {Ref }}$.

$P_{\mathrm{INF}}=$ McCARD/SCAN power by infinite medium spectrum.

$P_{\mathrm{CRI}}=\mathrm{McCARD} / \mathrm{SCAN}$ power by critical spectrum.

${ }^{*}$ Diff. $_{\text {INF }}(\%)=\left(P_{\text {INF }}-P_{\text {Ref }}\right) / P_{\text {Ref }} \times 100$, and ${ }^{* *}$ Diff. ${ }_{\text {CRI }}(\%)=\left(P_{\text {CRI }}-P_{\text {Ref }}\right) / P_{\text {Ref }} \times 100$. 


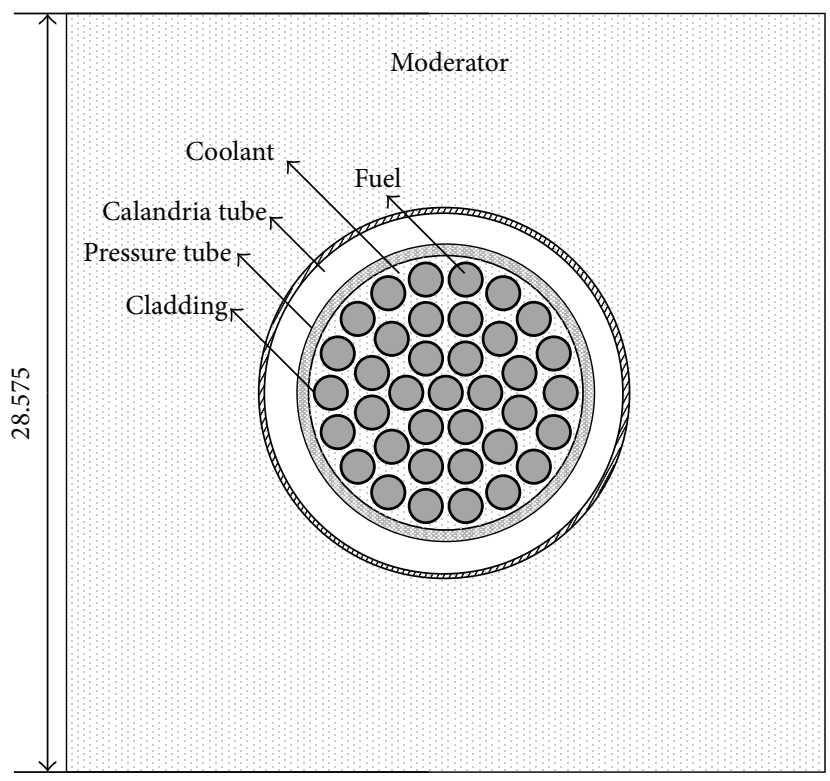

Reflective boundary conditions

FIgURE 5: Standard lattice cell model.

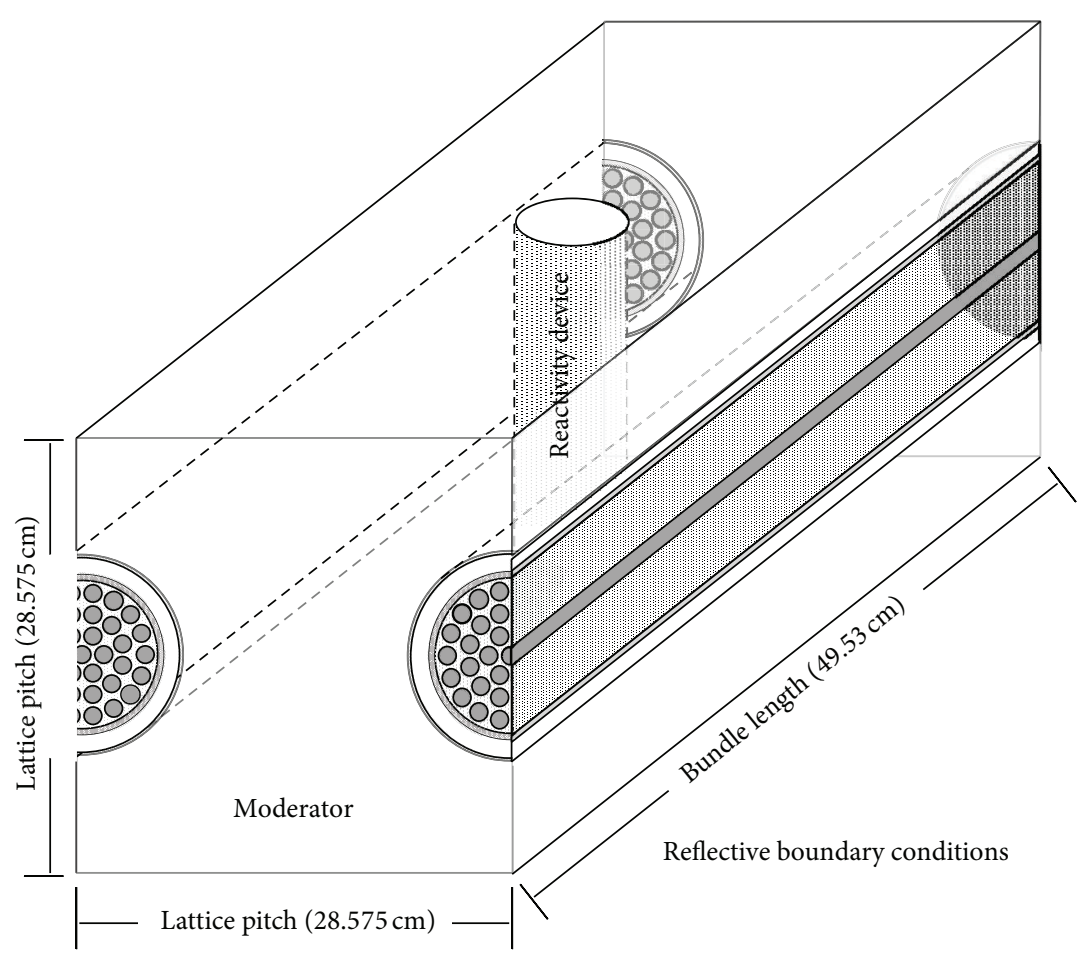

FIGURE 6: McCARD supercell model.

McCARD calculations are $0.39 \%, 0.83 \%$, and $1.36 \%$ and those with the IMS-weighted two-group cross sections $0.36 \%$, $0.80 \%$, and $1.46 \%$ for (i) the no-control-device core, (ii) the LZC-50\% fill core, and (iii) the all-ADJ-in core, respectively. Note also that the maximum channel power errors of the SCAN predictions to the reference McCARD calculations are $2.33 \%, 2.18 \%$, and $3.25 \%$ for the core (i), (ii), and (iii), respectively.

\section{Conclusion}

The above results show that not only do the CS-weighted twogroup constants generated by the $\mathrm{B}_{1} \mathrm{MC}$ method compare well with those by WIMS-AECL but also core neutronics analysis for the hypothetical CANDU 6 core problems by the SCAN calculation with the $B_{1} M C$ method generated two-group constants agrees well with that by the whole 
core reference McCARD calculation. Therefore, it is safely concluded that the $\mathrm{B}_{1}$ MC method is well qualified as a two-group constant generator for the standard unit lattice cell and the supercell and therefore it can serve a valuable alternative to its deterministic counterparts for the neutronics analysis of CANDU 6 reactors. The striking advantage of the $\mathrm{B}_{1} \mathrm{MC}$ method as a two-group constant generator for the neutronics analysis of CANDU-PHWR is its inherent capability to utilize the continuous-energy cross section library data and to model the geometrical heterogeneity of the fuel bundle cells-particularly those with control devicesexactly as they are. In this study, the CANDU-PHWR analysis utilized for the qualification test of the $\mathrm{B}_{1} \mathrm{MC}$ method has been confined to the hypothetic core problems. Further tests for the qualification of the $B_{1} M C$ method will be made in terms of realistic CANDU 6 reactor core problems.

\section{Conflict of Interests}

The authors declare that there is no conflict of interests regarding the publication of this paper.

\section{Acknowledgment}

This work was supported by the National Research Foundation of Korea Grant funded by the Government of Republic of Korea (Ministry of Science, ICT, and Future Planning) (no. NRF-2012M2A8A4011779).

\section{References}

[1] H. J. Shim, J. Y. Cho, J. S. Song, and C. H. Kim, "Generation of few group diffusion theory constants by Monte Carlo code," Transactions of the American Nuclear Society, vol. 99, pp. 343345, 2008.

[2] H. J. Park, H. J. Shim, H. G. Joo, and C. H. Kim, "Generation of few-group diffusion theory constants by Monte Carlo code McCARD," Nuclear Science and Engineering, vol. 172, no. 1, pp. 66-77, 2012.

[3] M. Edenius and B. H. Forrsen, "CASMO-3 a fuel assembly burnup program user's manual," Tech. Rep. Studsvik/NFA-893, Rev. 2, Studsvik AB, 1992.

[4] R. J. J. Stammler, HELIOS Methods, Studsvik Scandpower, 2002.

[5] J. D. Irish and S. R. Douglas, "Validation of WIMS-IST," in Proceedings of the 23rd Annual Conference of Canadian Nuclear Society, Toronto, Canada, June 2002.

[6] H. J. Shim, B. S. Han, S. J. Jong, H. J. Park, and C. H. Kim, "McCARD: Monte Carlo code for advanced reactor design and analysis," Nuclear Engineering and Technology, vol. 44, no. 2, pp. 161-176, 2012.

[7] G. Marleau, A. Hebert, and R. Roy, "A user's guide for DRAGON version DRAGON_980911 release 3.03," Tech. Rep. IGE-174, Revision 4, Ecole Polytechnique de Montreal, 1998.

[8] M. Ovanes, D. A. Jenkins, F. Ardeshiri et al., "Validation of the RFSP-IST code against power-reactor measurements," in Proceedings of 22nd Annual Conference of Canadian Nuclear Society, Toronto, Canada, June 2001.
[9] A. R. Dastur and D. Buss, "MULTICELL-a 3-D program for the simulation of reactivity devices in CANDU reactors," AECL 7544, Atomic Energy of Canada Limited, 1983.

[10] I. S. Hong, C. H. Kim, B. J. Min, and H. C. Suk, "Development of CANDU-PHWR neutronics code SCAN," in Proceedings of the 7th International CANDU Fuel Conference, vol. 1, pp. 77-86, Canadian Nuclear Society, Kingston, Canada, September 2001. 


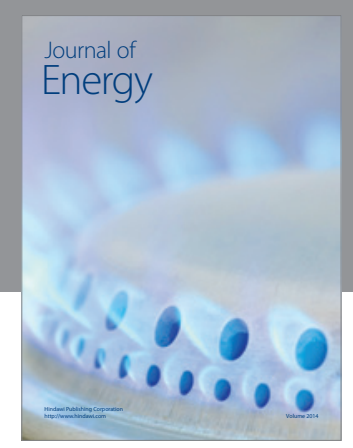

Journal of

Industrial Engineering
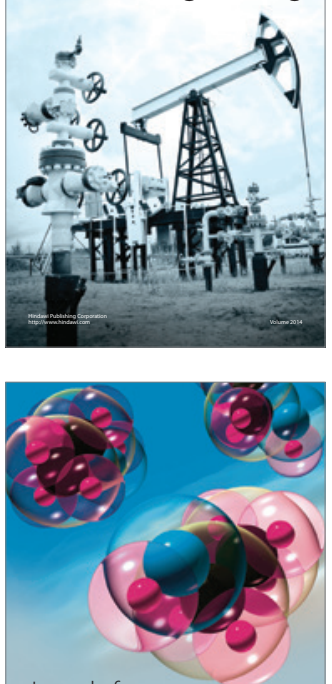

Fuels
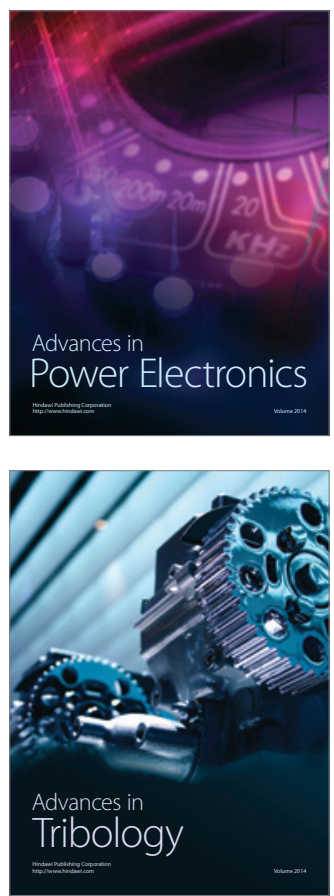

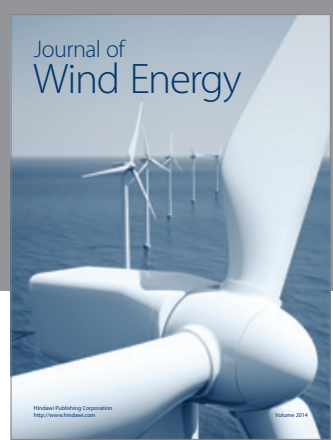

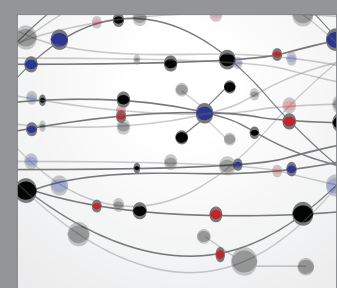

The Scientific World Journal

Submit your manuscripts at http://www.hindawi.com

Journal of

Structures
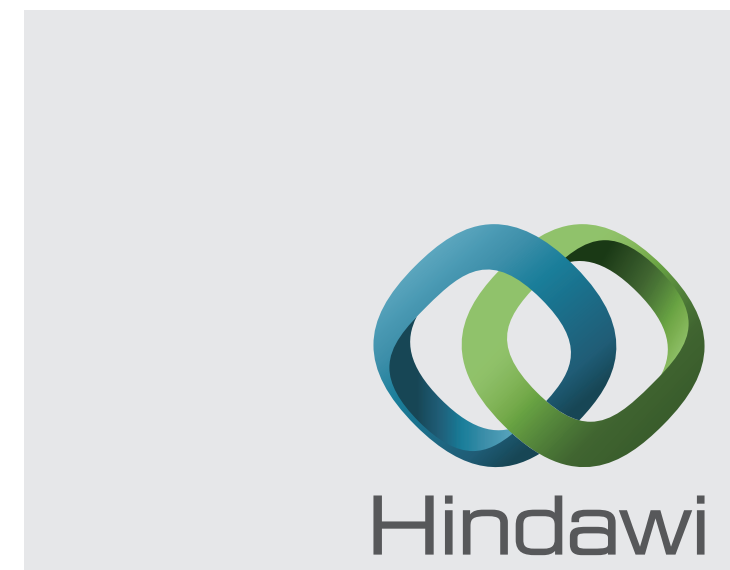

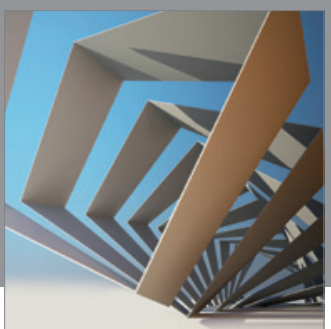

Rotating

Machinery
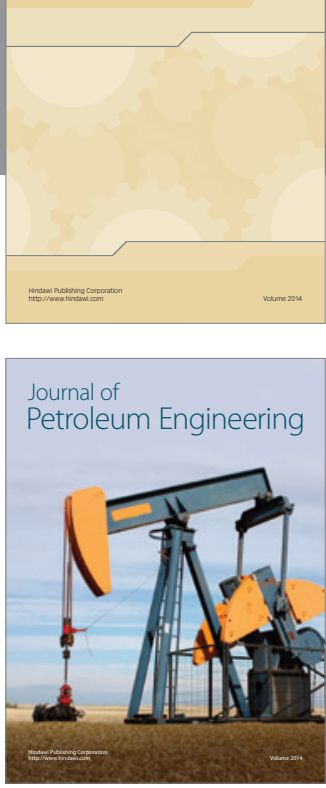

Journal of

Solar Energy
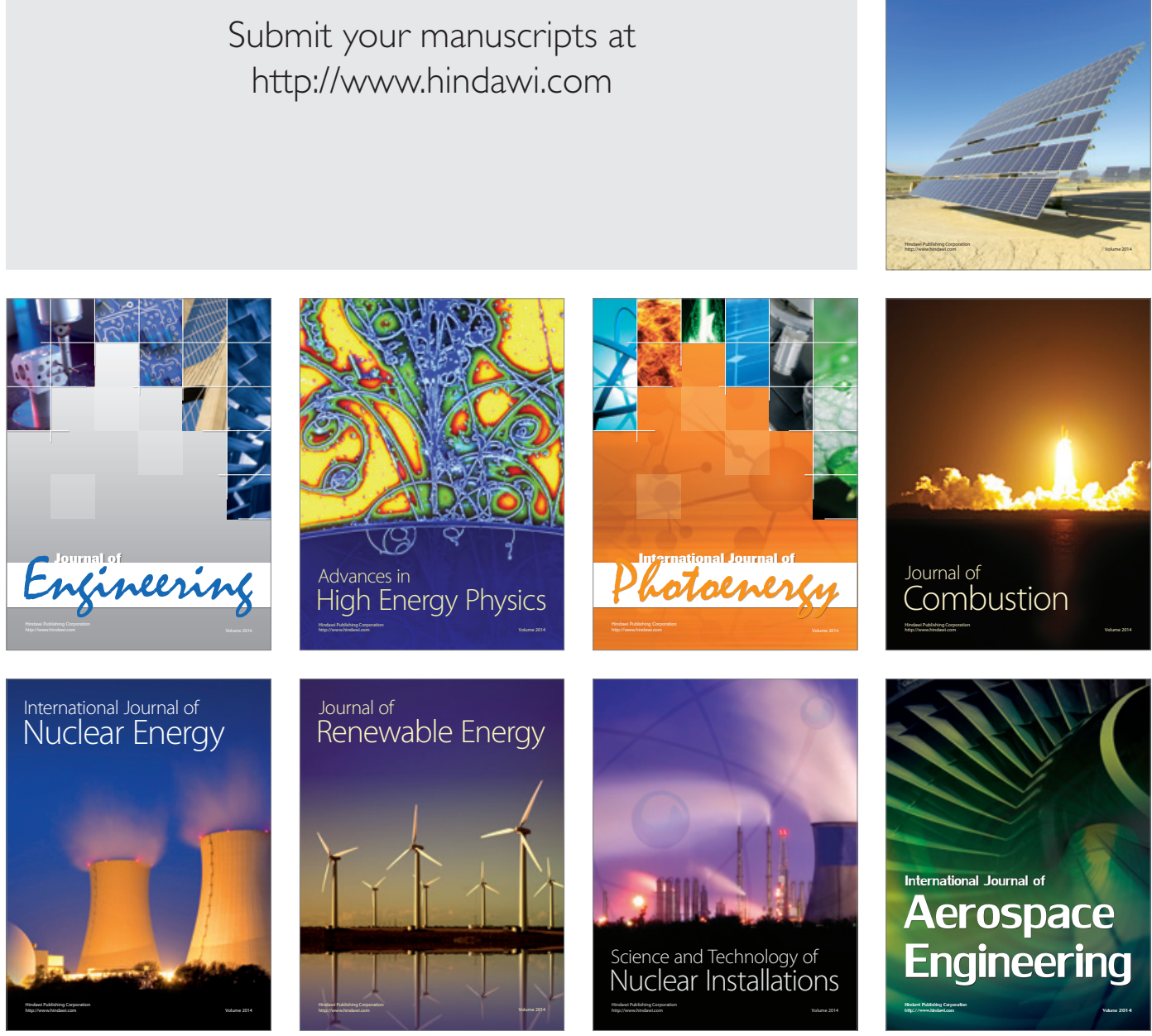